\title{
International Translational Research: Promise and Caution
}

\author{
Richard F. Catalano ${ }^{\mathrm{a}, *}$ and Harrie Jonkman ${ }^{\mathrm{b}}$ \\ ${ }^{a}$ University of Washington, USA \\ ${ }^{\mathrm{b}}$ Verwey-Jonker Institute, Utrecht, $N L$
}

A recent series on adolescent health in The Lancet described the success of concerted worldwide effort in infectious disease and the global investment in child health (Resnick, Catalano, Sawyer, Viner, \& Patton, 2012). As a result more children are surviving into adolescence and the world wide burden of disease has shifted to non-communicable diseases often due to behavior problems that begin during adolescence and add to the burden of disease during adolescence but also through the life course. The Institute of Medicine report on preventing mental and behavioral disorders, for example, demonstrated that mental, emotional and behavioural disorders like depression, substance abuse, and conduct disorders affect large numbers of young people which have a life-long psycho-social effect on the young people, their families, schools and communities (Institute of Medicine [IOM], 2009). Unicef also has recently targeted adolescence as the central phase for physical, mental, sexual, and reproductive health and promotes its social investment to end the cycle of poverty, to eliminate inequities and to secure a better future for children and young people (Unicef, 2011). The Worldbank gave a message to governments and policy makers to invest in young people for countries development (The Worldbank, 2006). All these documents call on international, national, and local leaders to

\footnotetext{
*Address for correspondence

Richard F. Catalano, Jr., University of Washington, USA. E-mail: catalano@uw.edu.
}

make prevention and promotion of health among youth top priority.

As described in the article by Catalano et al. (2012) of the Lancet-series, these behaviour problems are largely preventable. Wide ranging approaches have been found to be efficacious in multiple contexts, community, family, school, and individual approaches. These approaches have been found to be efficacious in high income and lower and middle income countries. Catalano and colleagues suggested that to reduce the burden of disease from these adolescent initiated behavior problems, the global challenge is to increase use of tested, efficacious prevention policies and programs while recognizing that communities and nations are different from one another and need to decide locally what policies and programs they use.

The Lancet prevention article by Catalano et al. (2012) suggests that research on translating these efficacious prevention approaches to a range of communities and countries is necessary. The issue is how to do this in a way that recognizes local conditions, but also recognizes that the policy or program active ingredients need to be preserved in order for the program to work. While there is evidence that efficacious interventions can be successfully replicated in different contexts, there is considerable debate regarding how to transfer programs to new settings. Some suggest that high fidelity is necessary to produce positive outcomes (Durlak \& DuPre, 2008; Elliott \& Mihalic, 2004), while others contend 
that adaptations are necessary to ensure that preventive interventions are culturally appropriate and relevant to the new population (Castro, Barrera Jr., \& Martinez Jr., 2005; Madon, Hofman, Kupfer, \& Glass, 2007; Tsarouk, Thompson, Herting, Walsh, \& Randell, 2007).

The Community that Care (CTC) prevention system was built using the research base for prevention science. It mobilizes community coalitions of stakeholders to prevent adolescent behavioural problems and is designed to build community capacity. Coalitions assess and prioritize local risk and protective factors through the CTC youth survey and plan and deliver adolescent health and community prevention by implementing tested and effective preventive programs to address elevated risks (Hawkins \& Catalano, 1992, 2005). A randomized trial involving 24 communities in the USA showed positive results on prevention of adolescent drug use and delinquency and showed outcome effects after four years of implementing the program on drug use (tobacco and underage drinking), on delinquency and violence as well as on certain risk factors (Brown, Hawkins, Arthur, Briney, \& Fagan, 2011; Feinberg, Jones, Greenberg, Osgood, \& Bontempo, 2010; Hawkins et al., 2009). Sustained effects could be found two years after the end of project funds to support the intervention (Hawkins et al. 2011).

The CTC-prevention system is introduced to communities in the Netherlands with the same core elements as was used in the USA expecting the same initiation and development outcomes on youngsters in the communities after introduction of the program (Jonkman et al., 2008; Steketee et al., 2012). Different from the U.S. American studies no influence of the intervention could be detected in the effect study (see Jonkman, Aussems, Steketee, Boutellier, \& Cuijpers, 2015). Why was this the case, and what can we learn about the prospects of international prevention translational research?

Translational research can be fostered by identification of the core elements of efficacious interventions - the content, activities, and modes of delivery that best represent their underlying logic and causal mechanisms (Elliott \& Mihalic, 2004; Fixsen, Naoom, Blase, Friedman, \& Wallace, 2005; Rotheram-Borus, Ingram, Swendeman, \& Flannery, 2009). Jonkman and colleagues' implementation included core elements of CTC. Coalitions were mobilized, training was provided in prevention science and prevention assessment of outcomes risk and protective factors. The CTC survey was translated to Dutch and local adolescents were assessed, and local profiles were constructed to determine priority risk and protective factors to address. However, matching these local priorities to tested, effective prevention programs, a core element of CTC, was not possible due to the lack of rigorously tested programs available in the Dutch context. CTC relies on a rich array of tested prevention programs that can be chosen to address local need. This points out another conclusion of the Lancet prevention article by Catalano et al. (2012) that most of the testing of preventive interventions has been conducted in high income counties, primarily in the U.S. To achieve the promise of prevention of behavior problems globally, the first step may be that specific programs need to be adapted (translated and fit into the service delivery system) and be tested in rapid trials. Processes like the ADAPT-ITT might be utilized to translate tested interventions to local contexts (Wingood \& DiClemente, 2008). This adaptation process must consider core elements of the intervention and adaptations to fit into the local culture (often cultural elements are expressed in prevalence of risk and protective factors), communication methods that local populations can relate to, recruitment to the intervention localized (Catalano et al., 1993), and delivery made compatible with local resources and condition. Once these adaptations are made small randomized trials (with adequate, but minimal numbers to achieve power to detect effects) with efficient measurement of relevant outcomes might be conducted to provide local evidence of the impact of adapted interventions. Programs like CTC which are designed to enhance the use of tested, effective prevention strategies might need to be delayed until the local prevention evidence base is constructed.

Since there is an array of tested prevention programs, a question that arises is which tested, effective programs to start in different contexts. This might be answered locally by assessing risk and protection through national or regional surveys using instruments like the CTC youth survey. The CTC survey, which has been translated and used in a number of contexts with reliable and valid results, is designed to provide a profile of outcomes, risk and protective factors that could point to national or regional priority needs for risk reduction and protective factor enhancement that could be matched to existing tested, effective prevention programs which could become priority targets for translational research.

These global efforts within countries are one way to rapidly build up the local evidence base for prevention, since they can adapt interventions tested in other contexts which have already conducted considerable developmental research and testing. This is also likely to 
build up the global experience in conducting controlled trials.

Over the last 30 years, prevention science has developed a strong research base in epidemiology and preventive trials. Only recently has the call for tested prevention programs spread beyond high income countries and most prevention trials have been conducted in the U.S. Catalano and colleagues (2012) pointed out that one solution to the global challenge to increase use of tested, efficacious prevention policies and programs globally was to build capacity and interest among researchers, governments, and practitioners. Both translational prevention research and building experience with trials would contribute to local capacity and interest in prevention science. Jonkman and colleagues (2015) point out that the researchers did not have adequate capacity or funding to control many of the critical processes, funding for interventions, and the ability to prevent contamination of control communities. Further, a lack of appreciation for random assignment to conditions was also a challenge to the scientific integrity of this study. These challenges suggest that a first step may be conducting translational research trials of efficacious prevention programs. Once expertise in trials and prevention program delivery is built through these best bets, it may be more likely that indigenous prevention programs might be readied or developed for testing, and over the medium term create more efficacious prevention programs to reduce global burden. Further, the experience with trials and program adaptation will increase the capacity of countries to develop and test prevention programs which respond to unique local challenges, leveraging the impact of prevention. The investment and experience in child health has proceeded in a like manner, using translational research to understand how to deliver and adapt tested innovations to increase maternal health and reduce infant mortality. International funding bodies should invest heavily in this type of translational research, to address the burden of behavior problems on adolescent and adult health.

\section{References}

Brown, E. C., Hawkins, J. D., Arthur, M. W., Briney, J. S., \& Fagan, A. A. (2011). Prevention service system transformation using communities that care. Journal of Community Psychology, 39, 183-201.

Castro, F. G., Barrera Jr., M., \& Martinez Jr., C. R. (2005). The cultural adaptation of prevention interventions: Resolving tensions between fidelity and fit. Prevention Science, 5, 41-46.
Catalano, R. F., Fagan, A. A., Gavin, L., Greenberg, M. T., Erwin, C. E., Ross, D. A.,... Shek, D. T. L. (2012). Worldwide application of prevention science in adolescent health. The Lancet, 379, 16531664.

Catalano, R. F., Hawkins, J. D., Krenz, C., Gillmore, M., Morrison, D., Wells, E., \& Abbott, R. (1993). Using research to guide culturally appropriate drug abuse prevention. Journal of Consulting and Clinical Psychology, 61, 804-811.

Durlak, J. A., \& DuPre, E. P. (2008). Implementation matters: A review of research on the influence of implementation on program outcomes and the factors affecting implementation. American Journal of Community Psychology, 41, 327-350.

Elliott, D. S., \& Mihalic, S. (2004). Issues in disseminating and replicating effective prevention programs. Prevention Science, 5 , 53.

Feinberg, M. E., Jones, D., Greenberg, M. T., Osgood, D. W., \& Bontempo, D. E. (2010). Effects of the Communities that Care model in Pennsylvania on youth risk and problem behaviors. Prevention Science, 11, 163-171.

Fixsen, D. L., Naoom, S. F., Blase, K. A., Friedman, R. M., \& Wallace, F. (2005). Implementation research: A synthesis of the literature. No. FMHI Publication \#231. Tampa, FL: University of South Florida, Louis de la Parte Florida Mental Health Institute, The National Implementation Research Network.

Hawkins, J. D., \& Catalano, R. F. (1992). Communities that Care: Action for drug abuse prevention. San Fransico: Jossey-Bas, Publishers.

Hawkins, J. D., \& Catalano, R. F. (2005). Investing in your community's youth: An introduction to the Communities that Care system. South Deerfield, MA: Channing Bete.

Hawkins, J. D., Oesterle, S., Brown, E. C., Arthur, M. W., Abbott, R. D., Fagan, A. A., \& Catalano, R. F. (2009). Results of a type2 translational research trial to prevent adolescent drug use and delinquency. Archives of Pediatric and Adolescent Medicine, 163, 789-798.

Hawkins, J. D., Oesterle, S., Brown, C. E., Monahan, K. C., Abbott, R. D., Arthur, M. W., \& Catalano, R. F. (2011). Sustained decreases in risk exposure and youth problem behaviors after installation of the communities that care prevention system in a randomized trial. Archives of Pediatric and Adolescent Medicine, 2011. doi: 10.1001/archpediatrics.2011.183.

Institute of Medicine (IOM) (2009). Report on preventing mental, emotional and behavioral disorders among young people: Progress and possibilities. Institute of Medicine: Washington.

Jonkman, H., Haggerty, K., Steketee, M., Fagan, A., Hanson, K., \& Hawkins, J. D. (2008). Communities that Care, core elements and context: Research of implementation in two countries. Social Development Issues, 30, 42-58.

Jonkman, H., Aussems, C., Steketee, M., Boutellier, H., \& Cuijpers, P. (2015) Prevention of problem behaviours among adolescents: The impact of the Communities that Care strategy in the Netherlands (2008-2011). International Journal of Developmental Science, 9 , 37-52.

Madon, T., Hofman, K. J., Kupfer, L., \& Glass, R. I. (2007). Implementation science. Science, 318, 1728-1729.

Resnick, M. D., Catalano, R. F., Sawyer, S. M., Viner, R. M., \& Patton, G. C. (2012). Comment: Seizing the opportunities of adolescent health. The Lancet, 379, 1564-1567.

Rotheram-Borus, M. J., Ingram, B. L., Swendeman, D., \& Flannery, D. (2009). Common principles embedded in effective adolescent HIV prevention programs. Aids and Behavior, 13, 387-398. 
Steketee, M., Jonkman, H., Mak, J., Aussems, C., Huygen, A. \& Roeleveld, W. (2012). [Communities that Care in Nederlandse steden]. Resultaten van een vierjarig experimenteel onderzoek. Utrecht: Verwey-Jonker Instituut.

The Worldbank (2006). World development report 2007. Development and the next generation. Washington DC: The Worldbank.

Tsarouk, T., Thompson, E. A., Herting, J. R., Walsh, E., \& Randell, B. (2007). Culturally specific adapation of a prevention intervention: An international collaborative research project. Addictive Behaviors, 32, 1565-1581.

Unicef (2011). The state of the world's children 2011. Adolescence: An age of opportunity. New York: Unicef.

Wingood, G. M., \& DiClemente, R. J. (2008). The ADAPT-ITT model: A novel model of adapting evidence based HIV interventions. JAIDS, 47 (S1), 40-46.

\section{Bio Sketches}

Richard F. Catalano, Jr., Ph.D. Social Development Research Group-Director and Bartley Dobb Professor for the Study of Prevention of Violence.

Harrie Jonkman, Ph.D. Senior at the Verwey Jonker Institute. Their work focus on risk and protective factors for positive and problem behaviour, designing and evaluating effective programs, using this knowledge to understand and improve prevention in states and communities. 